\title{
Elucidating the Barriers on Direct Water Splitting: Key Role of Oxygen Vacancy Density and Coordination over $\mathrm{PbTiO}_{3}$ and $\mathrm{TiO}_{2}$
}

\author{
Ersen Mete,* Şinasi Ellialtıoğlu, Oguz Gulseren, and Deniz Uner* \\ Cite This: J. Phys. Chem. C 2021, 125, 1874-1880 \\ Read Online
}

ABSTRACT: In this work, using the state-of-the-art firstprinciples calculations based on density functional theory, we found that the concentration and coordination of surface oxygen vacancies with respect to each other were critical for the direct water-splitting reaction on the (001) surfaces of $\mathrm{PbTiO}_{3}$ and $\mathrm{TiO}_{2}$. For the water-splitting reaction to happen on $\mathrm{TiO}_{2}$-terminated surfaces, it is necessary to have two neighboring $\mathrm{O}$ vacancies acting as active sites that host two adsorbing water molecules. However, eventual dissociation of $\mathrm{O}-\mathrm{H}$ bonds is possible only in the presence of an additional nearest-neighbor $\mathrm{O}$ vacancy. Unfortunately, this necessary third vacancy inhibits the formation of molecular hydrogen by trapping the dissociated $\mathrm{H}$ atoms on $\mathrm{TiO}_{2}-$ terminated surfaces. Formation of up to three $\mathrm{O}$ vacancies is

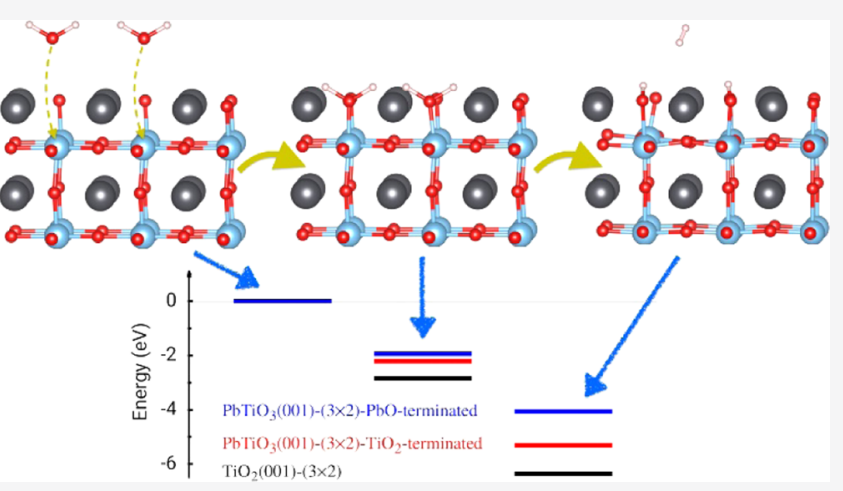
energetically less costly on both terminations of $\mathrm{PbTiO}_{3}(001)$ surfaces compared with those on $\mathrm{TiO}_{2}$; the presence of $\mathrm{Pb}$ leads to

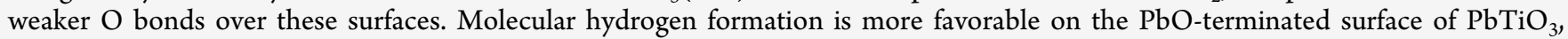
requiring only two neighboring oxygen vacancies. However, the hydrogen molecule is retained near the surface by weak van der Waals forces. Our study indicates two barriers leading to low productivity of direct water-splitting processes. First and foremost, there is an entropic barrier imposed by the requirement of at least two nearest-neighbor $\mathrm{O}$ vacancies, sterically hindering the process. Furthermore, there are also enthalpic barriers of formation on $\mathrm{TiO}_{2}$-terminated surfaces or removal of $\mathrm{H}_{2}$ molecules from the $\mathrm{PbO}$ terminated surface. The requirement dictating three nearest-neighbor oxygen vacancies for hydrogen evolution is also consistent with the chemical intuition: The nearest neighbor of the formed hydrogen should be reduced enough to inhibit spontaneous oxidation under ambient conditions.

\section{INTRODUCTION}

Understanding and finding a route toward an efficient watersplitting reaction is not only important for energy and technological applications ${ }^{1}$ but also essential from the fundamental point of view. Several different model systems like titania or perovskite surfaces have been proposed and investigated for this purpose. ${ }^{2-4}$ Existing routes for hydrogen production from renewable energy include (i) using renewable electricity for electrolysis of water, ${ }^{5}$ (ii) using concentrated solar energy for steam and/or carbon dioxide reforming of methane, ${ }^{6,7}$ and (iii) photoassisted catalytic or electrochemical water splitting. ${ }^{8}$ If proven successful, photo(electro)chemical production of hydrogen is the most attractive due to the simplicity of the process and the potential this simple process offers. 9

Both direct photo-electrochemical and photocatalytic water splitting suffer from two problems. The first problem is the low production rates and photon conversion efficiencies. The second problem, which is also directly related to the first problem, is the difficulty with which the structures release oxygen for a complete catalytic cycle. In photo-electrochemical processes, the bottleneck is the oxygen release. The thermodynamic driving forces under ambient conditions are toward water; hence, once hydrogen and oxygen molecules are produced, their contact must be avoided to inhibit spontaneous combustion. This condition is present in electrochemical cells in which the anode and cathode are connected through a circuit and an electrolyte, but the produced hydrogen and oxygen are collected separately. Still, in the electrochemical systems, the bottleneck is the oxygen evolution reaction. ${ }^{10}$ The current consensus for the prevailing mechanism of oxygen evolution in oxides or perovskites is through the formation of oxygen vacancy. The subsequent steps of this process depend on several factors including the nature of the oxide material.

Received: October 26, 2020

Revised: December 20, 2020

Published: January 19, 2021 
On the contrary, photocatalytic hydrogen production is carried out in slurry-type suspended solid catalysts in solvents. Well-mixed conditions, needed for an efficient reaction, also provide the environment for the immediate back oxidation of hydrogen to water. These processes can be inhibited by a careful design of the photoreactor or designing catalysts where oxygen evolution and hydrogen evolution are delegated to different facets of the same crystallite. ${ }^{9}$ Despite the efforts, hydrogen production rates are within a few $\mathrm{mmol} /(\mathrm{h} \cdot \mathrm{g})$ catalyst as revealed by a thorough review of the literature, accompanied by the machine learning approaches. ${ }^{11}$

The dilemma in all of these processes resides in the thermochemical bulk properties of the semiconductor oxides. The inherent photocatalytic activity of the semiconductor oxides depends on the capacity of bond breaking by the interaction of the oxide with electromagnetic radiation. The absorption of the electromagnetic radiation with the proper wavelength results in the creation of $\mathrm{e}^{-}-\mathrm{h}^{+}$pairs. The chemical nature of these $\mathrm{e}^{-}-\mathrm{h}^{+}$pairs depends on the oxide itself. For $\mathrm{TiO}_{2}$ as an example, upon irradiation, $\mathrm{e}^{-}$finds a place on the $\mathrm{Ti}$ atom, changing the oxidation state from +4 to +3 , while the bond between the oxygen and $\mathrm{Ti}$ atoms are broken. In other words, "softness" of the bonds between oxygen and metal atoms is required for photon absorption, and, on the other hand, softness is the reason that the formed hydrogen can interact with these bonds for oxidation. The chemical potential of the hydrogen formed is sufficient to reduce the lattice oxygen.

One final barrier in the efficient production of hydrogen is to remove the hydrogen formed by water splitting that is accommodated on the solid. Hydrogen can be accommodated in the form of spilled over hydrogen, which can be held around the surface or in the bulk. ${ }^{12}$ The limitations can also be due to kinetic metastabilities dictated by a low surface coverage of hydrogen atoms. The spatial separation may inhibit the formation of dihydrogen for desorption, hence lowering the productivity.

The objective of this study is to search the limits of the water-splitting reaction over $\mathrm{TiO}_{2}$ and $\mathrm{PbTiO}_{3}$ through firstprinciples methods. We determined the barriers to the formation and removal of molecular hydrogen on titania and lead titanate. We started our analysis by assuming that oxygen vacancies are available, acting as two neighboring active sites hosting two water molecules. We examined the possibility of water dissociation depending on the coordination and clustering of surface $\mathrm{O}$ vacancies by $\mathrm{ab}$ initio methods.

\section{METHODS}

Density functional theory (DFT) calculations were performed using VASP $^{13}$ within the framework of the projector augmented-wave (PAW) method $^{14}$ based on plane-wave expansion of single-particle states up to an energy cutoff value of $400 \mathrm{eV}$. The exchange and correlation effects were taken into account in the calculations by the SCAN (strongly constrained and appropriately normed) ${ }^{15}$ meta-GGA (semilocal meta-generalized gradient approximation) density functional. The SCAN functional lifts the overbinding in oxide materials, which is common to GGA functionals. ${ }^{16}$ The shortcoming of semilocal XC functionals in describing the electronic structures of materials associated with selfinteraction (SI) can be overcome by either admixing the Hartree-Fock exchange with local density approximation (LDA)-based exchange terms or supplementing on-site
Coulomb interaction for localized electrons, a method known as $\mathrm{LDA}+U$. In particular, the transition-metal oxides with partially occupied d-states were erroneously predicted to be metallic by semilocal XC functionals. A proper description of both defect-related gap states and the band gaps can be obtained by imposing a suitably chosen $U$ parameter for Ti $3 \mathrm{~d}$ states. Recently, the use of $U$ parametrization for $\mathrm{O} 2 \mathrm{p}$ states in oxides was shown to enhance the energetics and forces to match those of hybrid functionals. ${ }^{17}$ A systematic trace of the $U$-parameter space showed that defect-related electronic properties can be properly described when the SCAN functional is supplemented with parameters of $U=6$ for $\mathrm{Ti}$ $3 \mathrm{~d}$ and $U=3$ for $\mathrm{O} 2 \mathrm{p}$ states. The calculated electronic band gaps of pristine $\mathrm{PbTiO}_{3}$ and $\mathrm{TiO}_{2}$ are $2.53 \mathrm{eV}\left(\right.$ exp. $2.5 \mathrm{eV}^{18}$ ) and $2.77 \mathrm{eV}\left(\exp .3 .1 \mathrm{eV}^{18}\right)$, respectively. A $4 \times 8 \times 1 k$-point mesh was used for Brillouin zone integrations. Atomic coordinates were fully relaxed by imposing a convergence criterion of $10^{-2} \mathrm{eV} /$ atom for the minimization of HellmannFeynman forces on each atom in every spatial cartesian direction.

Both $\mathrm{PbO}$ and $\mathrm{TiO}_{2}$ terminations of $\mathrm{PbTiO}_{3}(001)-(3 \times 2)$ and $\mathrm{TiO}_{2}(001)-(3 \times 2)$ surfaces were modeled as slabs that were constructed from their bulk phases, including 11 atomic layers along the [001] direction, to ensure the convergence of surface energies, as suggested previously. ${ }^{19} \mathrm{~A}$ vacuum region with a thickness of $12 \AA$ along the surface normal was introduced in the computational supercells to avoid any unphysical interaction between the periodic images of the slabs. Full-geometry optimizations were performed without freezing any of the atoms to their bulk positions and without imposing symmetry. In addition, the postprocessing of the calculated data was needed to determine the charge accumulation around each atom, which was carried out using the Bader algorithm. ${ }^{20}$

\section{RESULTS AND DISCUSSION}

There are basically two possible terminations for the lowest index (001) plane of $\mathrm{PbTiO}_{3}$. These are the $\mathrm{PbO}$ and $\mathrm{TiO}_{2}$ surface layers. The latter has the lowest surface energy among all possible cleavages including higher-index planes. ${ }^{21}$ Therefore, the (001) surface with $\mathrm{TiO}_{2}$-termination is the most stable surface of $\mathrm{PbTiO}_{3}$. When there are oxygen deficiencies on the surface, $\mathrm{TiO}_{2}$-termination energetically becomes even more favorable than the $\mathrm{PbO}$ termination. ${ }^{22}$

Water-splitting reactions, whether thermochemical, electrochemical, or photocatalytic, involve reduction of oxide materials through an energy source such as heat, ${ }^{23,24}$ electrons, or photons. Therefore, oxygen vacancies play an important role in capturing and splitting of water molecules. ${ }^{25-28}$ We explored the necessary requirements for the direct splitting of water molecules via surface oxygen vacancies on $\mathrm{PbTiO}_{3}$ and $\mathrm{TiO}_{2}$. The conditions depend not only on the material but also on the termination due to different surface chemical reactivities.

The $\mathrm{TiO}_{2}$-terminated top layer of $\mathrm{PbTiO}_{3}$ mimics titania surfaces and provides a setting for a variety of chemical reactions. For instance, molecular adsorbates tend to exhibit similar adsorption characteristics to those on the anatase (001) surface. ${ }^{29,30}$ Bonds usually form between their tail oxygens and surface Ti atoms. This looks like a continuation of the bulklike $\mathrm{TiO}_{2}$ structure. In an experimental realization, the surface might have oxygen vacancies that leave excess charge around the nearest-neighbor $\mathrm{Ti}$ atoms. Such vacancy sites become 
more active in capturing molecules, leading to relatively stronger binding.

The energy required to remove an oxygen atom from the surface is $3.86 \mathrm{eV}$ on the PbO-terminated and $3.96 \mathrm{eV}$ on $\mathrm{TiO}_{2}$-terminated surface. It is energetically $0.1 \mathrm{eV}$ more favorable on the $\mathrm{PbO}$-terminated surface. The formation energy of an oxygen vacancy on the surface of titania is $4.39 \mathrm{eV}$ (Table 1). We explored necessary conditions for hydrogen

Table 1. Formation Energies (in eV) of Nearest-Neighbor Surface Oxygen Vacancies Calculated Using the SCAN XC Functional

\begin{tabular}{lccc}
\multicolumn{1}{c}{ surface model } & $1 \mathrm{O}_{\text {vac }}$ & $2 \mathrm{O}_{\text {vac }}$ & $3 \mathrm{O}_{\text {vac }}$ \\
$\mathrm{TiO}_{2}(001)-(3 \times 2)$ & 4.39 & 9.77 & 15.39 \\
$\mathrm{PbTiO}_{3}(001)-(3 \times 2) \mathrm{TiO}_{2}$-terminated & 3.96 & 7.39 & 11.49 \\
$\mathrm{PbTiO}_{3}(001)-(3 \times 2) \mathrm{PbO}^{- \text {terminated }}$ & 3.86 & 8.12 & 11.37 \\
\hline
\end{tabular}

molecule formation as a result of oxygen vacancy-induced splitting of water on the surfaces. To examine the possibility of splitting two adjacent water molecule adsorbates into their hydrogen atoms and hydroxyl groups on the surfaces, we considered a number of configurations. As a starting point, the minimum condition appears to be the availability of adjacent surface oxygen vacancies that act as an adsorption center. The adsorption of two water molecules at the two adjacent oxygen vacancy sites allows close proximity for the nearest-neighbor hydrogen atoms that face each other in the lateral direction. The energy cost of formation of two and three neighboring surface oxygen vacancies, in Table 1 , increases depending on the material and surface termination; the formation energy per vacancy appears to slightly fluctuate around the value of a single vacancy.

In the case of $\mathrm{TiO}_{2}$ termination on both titania and lead titanate, the adsorption geometry of two water molecules at the two neighboring oxygen vacancy sites is shown in Figures $1 \mathrm{~b}$ and $2 \mathrm{~b}$, respectively. Formation of $\mathrm{Ti}-\mathrm{O}$ bonds as a result of charge transfer from the surface $\mathrm{Ti}$ to oxygen atom due to water adsorption leads to weakening of $\mathrm{O}-\mathrm{H}$ bonds within the water molecule. Bader analysis, as a comparison of the total charge on the substrate before and after adsorption, show a charge transfer of 1.43 and 1.39 electrons from the titania and $\mathrm{TiO}_{2}$-terminated lead titanate slabs, respectively. This type of surface oxidation and atomistic configuration solely is not enough to achieve water splitting. The DFT calculations indicated that there is a need for an additional oxygen vacancy that must also be adjacent to both of the adsorption sites, as shown in Figures $1 \mathrm{~b}$ and $2 \mathrm{~b}$. Adsorption of water on the surface loosens the $\mathrm{O}-\mathrm{H}$ bonds. Moreover, the water molecules slightly lean toward each other due to the absence of lateral $\mathrm{Ti}-\mathrm{O}$ bonds between them. Then, the distance between the $\mathrm{H}$ atoms facing each other becomes as small as 1.8 $\AA$ in the lateral direction due to the presence of the third oxygen vacancy. This site has to remain as a vacancy to ensure breaking the $\mathrm{O}-\mathrm{H}$ bonds. This mechanistic picture gives rise to the dissociation of $\mathrm{H}$ atoms from their parent water molecules, leaving the hydroxyl groups behind. The ratio of the clustering vacancies to the number of surface oxygens is $1 / 3$. When we considered a cluster of four neighboring surface oxygen vacancies, the geometry optimization resulted in a significant local distortion, which drives the reduced surface layer to undergo reconstruction. These kinds of defect-driven structural changes due to oxygen vacancies are known for the formation of black $\mathrm{TiO}_{2} .{ }^{31,32}$ Although the $\mathrm{H}$ atoms that were dissociated from their parent water molecules are close to each other, they did not form a $\mathrm{H}_{2}$ molecule. On $\mathrm{TiO}_{2}$ termination, these hydrogen atoms were, however, attracted to the adjacent oxygen vacancy sites, forming different adsorption geometries for titania and lead titanate due to differences in their chemical environment at the subsurface layers, as shown in Figures 1c and $2 \mathrm{c}$. One of the main differences is the undulation geometry of the $\mathrm{TiO}_{2}$ network of the top layer, which mechanistically plays an important role in the subsequent pathway of the dissociated $\mathrm{H}$ atoms. The $\mathrm{Ti}-\mathrm{Ti}$ separations on the surface layer are 3.79 and $3.89 \AA$ on $\mathrm{TiO}_{2}(001)$ and $\mathrm{PbTiO}_{3}(001)$, respectively. Energetically, the adsorption of a water molecule at a surface oxygen vacancy site is considerably stronger on titania. The calculated adsorption energies are -1.43 and $-1.11 \mathrm{eV}$ on $\mathrm{TiO}_{2}(001)$ and $\mathrm{TiO}_{2}$-terminated $\mathrm{PbTiO}_{3}(001)$, respectively. On the surface of titania, both of the $\mathrm{H}$ atoms were trapped at the vacancy site, reflecting a $\mathrm{Ti}-\mathrm{H}$ bonding character, as shown in Figure 1c. Bader charge analysis shows an accumulation of 1.66 electrons around this $\mathrm{H}$ atom, indicating partial filling of the 2 s orbital. In this geometry, the position of lower-lying $\mathrm{H}$ mimics surface-like oxygen coordination with the nearest-neighbor $\mathrm{Ti}$ atoms. On the surface of lead titanate, one of the $\mathrm{H}$ atoms is attracted to the oxygen vacancy, while the other $\mathrm{H}$ atom is weakly bound atop the nearest-neighbor surface $\mathrm{Ti}$ atom (Figure 2c). Bader charges are 1.66 and 1.58 electrons for these $\mathrm{H}$ atoms, respectively. Both $\mathrm{H}$ atoms were trapped on the surface layer, preventing the formation of $\mathrm{H}_{2}$. Then, breaking of the translational symmetry induced by these impurities on the
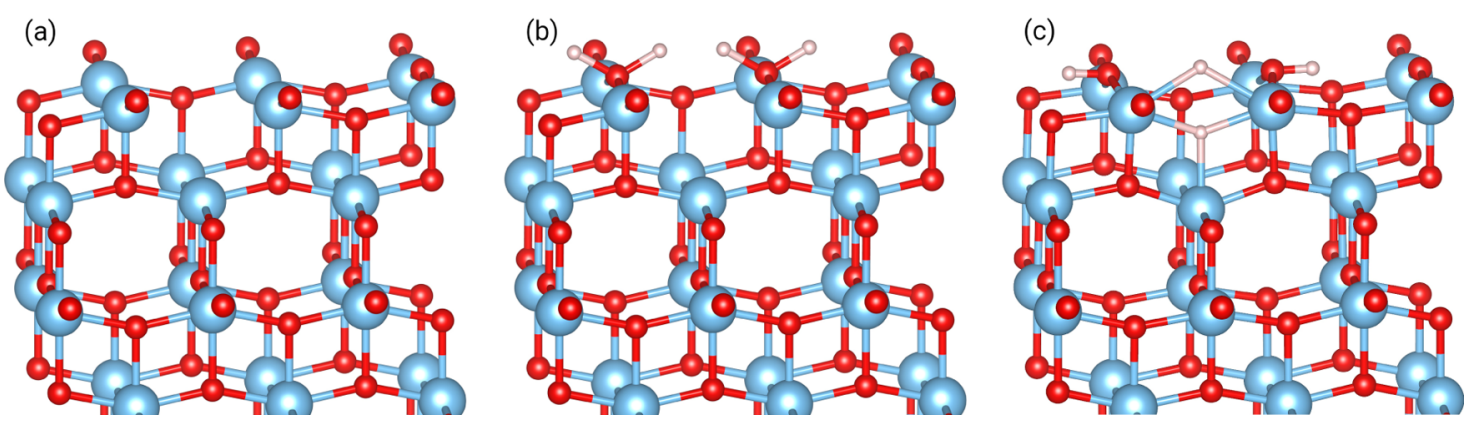

Figure 1. Schematics of $\mathrm{TiO}_{2}(001)-(3 \times 2)(\mathrm{a})$ with a cluster of three adjacent oxygen vacancies $\left(\mathrm{O}_{\text {vac }}\right)$ only and $\left(\mathrm{b}\right.$, c) with additional two $\mathrm{H}_{2} \mathrm{O}$ adsorbates at the neighboring oxygen vacancy sites in their corresponding initial and final geometries, respectively. Light blue ( $\mathrm{Ti})$, red $(\mathrm{O})$, and white $(\mathrm{H})$ colors are used for the ball-and-stick illustration of the model structure. 
(a)

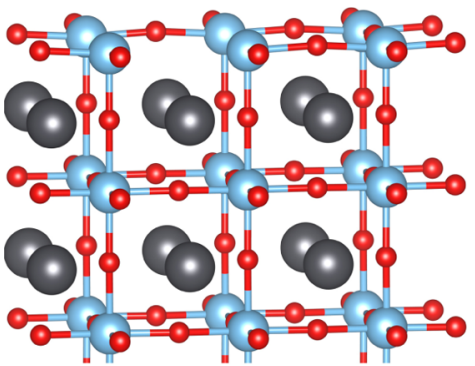

(b)

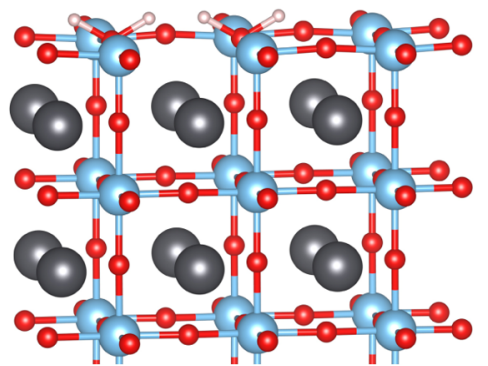

(c)

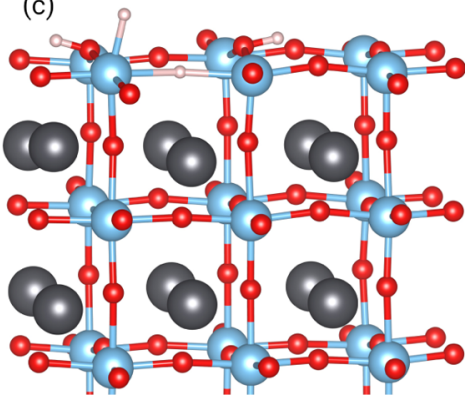

Figure 2. Schematics of $\mathrm{PbTiO}_{3}(001)-(3 \times 2) \mathrm{TiO}_{2}$-terminated (a) with three adjacent oxygen vacancies $\left(\mathrm{O}_{\text {vac }}\right)$ only and $(\mathrm{b}, \mathrm{c})$ with additional two $\mathrm{H}_{2} \mathrm{O}$ adsorbates at the neighboring oxygen vacancy sites in their corresponding initial and final geometries, respectively. Dark gray $(\mathrm{Pb})$, light blue $(\mathrm{Ti})$, red $(\mathrm{O})$, and white $(\mathrm{H})$ colors are used for the ball-and-stick illustration of the model structure.

(a)

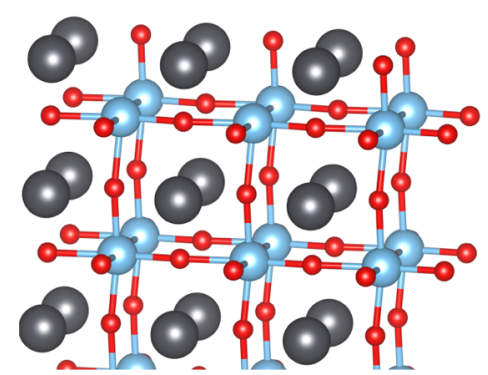

(b)

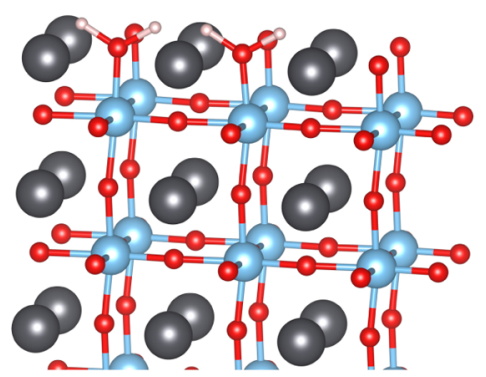

(c)

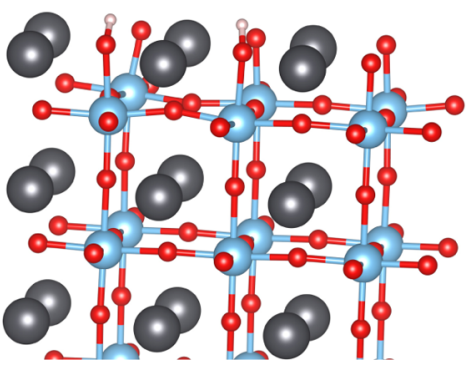

Figure 3. Schematics of $\mathrm{PbTiO}_{3}(001)-(3 \times 2)$ PbO-terminated $(\mathrm{a})$ with two adjacent oxygen vacancies $\left(\mathrm{O}_{\text {vac }}\right)$ only and $(\mathrm{b}, \mathrm{c})$ with additional two $\mathrm{H}_{2} \mathrm{O}$ adsorbates at the neighboring oxygen vacancy sites in their corresponding initial and final geometries, respectively.

surface layer influences the sublayer such that $\mathrm{Pb}$ atoms are distorted from their bulklike centroidal positions.

In the case of the $\mathrm{PbO}$-terminated surface of $\mathrm{PbTiO}_{3}$, when two water molecules are adsorbed on two nearest-neighbor oxygen vacancy sites, as shown in Figure 3b, they slightly lean toward each other and the $\mathrm{O}-\mathrm{H}$ bonds loosen as a result of charge transfer from the substrate to the adsorbate; Bader analysis gave a value of 0.85 electrons for lead titanate. Then, the $\mathrm{H}-\mathrm{H}$ distance becomes $1.8 \AA$ similar to the previous cases. The adsorption energy per water molecule at a surface oxygen vacancy site is $-0.97 \mathrm{eV}$, which is significantly lower in magnitude than those of $\mathrm{TiO}_{2}$ terminations. Another important difference of $\mathrm{PbO}$ termination from the $\mathrm{TiO}_{2}$ terminations is the coordination in the corresponding surface layers. The $\mathrm{Pb}-\mathrm{O}$ bonds are $2.54 \AA$ on $\mathrm{PbO}$ termination, while Ti-O bonds are $2.02 \AA$ on $\mathrm{TiO}_{2}$ termination. This indicates stronger coordination in the $\mathrm{TiO}_{2}$ network. These factors can be attributed to the dissociation of $\mathrm{H}$ atoms from their parent water molecules because of the attraction between the two hydrogen atoms in close proximity to each other. They form a $\mathrm{H}_{2}$ molecule, leaving the hydroxyl groups at the vacancy sites, because the required charge compensation is supplied by the $\mathrm{Ti}$ atoms that are exposed at the adsorption site. Bader charge analysis showed that the charge on each $\mathrm{H}$ atom that contributes to the covalent bonding in the hydrogen molecule is one electron, while the charge on the $\mathrm{H}$ atom of the hydroxyl group is zero. Then, the hydrogen molecule stays above the surface plane at a van der Waals distance $(2.90 \AA)$ due to the absence of an additional surface oxygen vacancy near the adsorption site, as shown in Figure 3c.

The energy level diagram for the pathway of water adsorption and dissociation on the oxygen-deficient surfaces is shown in Figure 4. Consistent with the calculated adsorption

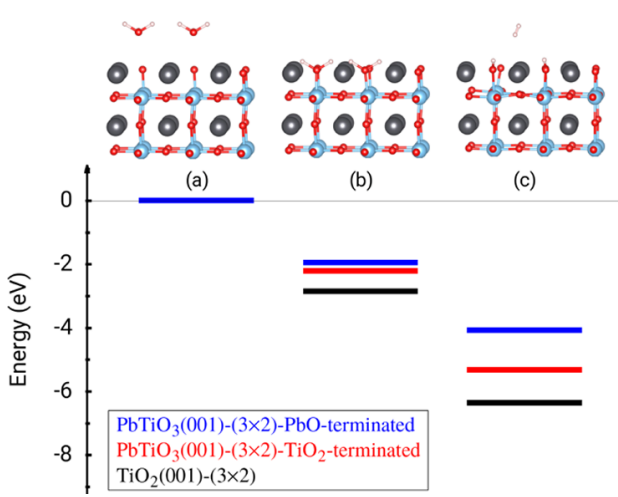

Figure 4. Energy level diagram of three stages of water splitting on $\mathrm{PbTiO}_{3}$ and $\mathrm{TiO}_{2}$ surfaces: (a) surface with adjacent oxygen vacancies and water molecules away from the surface, (b) water molecules get adsorbed, and (c) water splitting. Ball-and-stick illustration shown for $\mathrm{PbO}$-terminated surface of $\mathrm{PbTiO}_{3}$ as a representative.

energies, the first stage from (a) to (b) describes the separate adsorption of two water molecules on two nearest-neighbor oxygen vacancies. Binding of water on $\mathrm{TiO}_{2}$ is significantly stronger, which indicates the weakening of the lateral $\mathrm{Ti}-\mathrm{O}$ bonds associated with $\mathrm{Pb}$ atoms in the case of $\mathrm{PbTiO}_{3} \mathrm{~Pb}$ suppresses the undulation of the $\mathrm{Ti}-\mathrm{O}$ layer. The lateral $\mathrm{Ti}-\mathrm{O}$ bonds are 1.94 and $1.99 \AA$ in titania and lead titanate, respectively. For the second stage from (b) to (c), $\mathrm{TiO}_{2}-$ terminated surfaces appear to be energetically more favorable for water splitting. We note that dissociation of water did not take place on $\mathrm{TiO}_{2}$ terminations without an additional oxygen vacancy that connects the water adsorption sites. Therefore, at least a cluster of three oxygen vacancies is necessary on $\mathrm{TiO}_{2}$ terminations. However, dissociation of water did not end up 
(a)

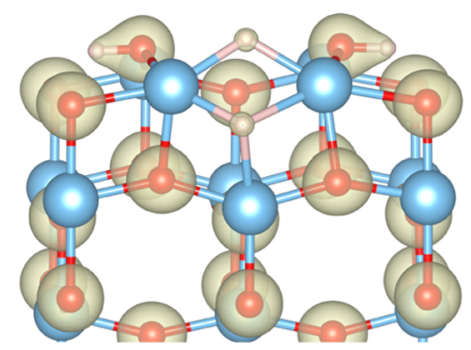

(b)

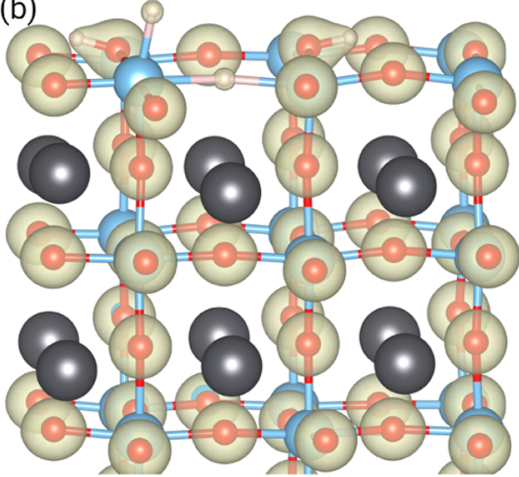

(c)
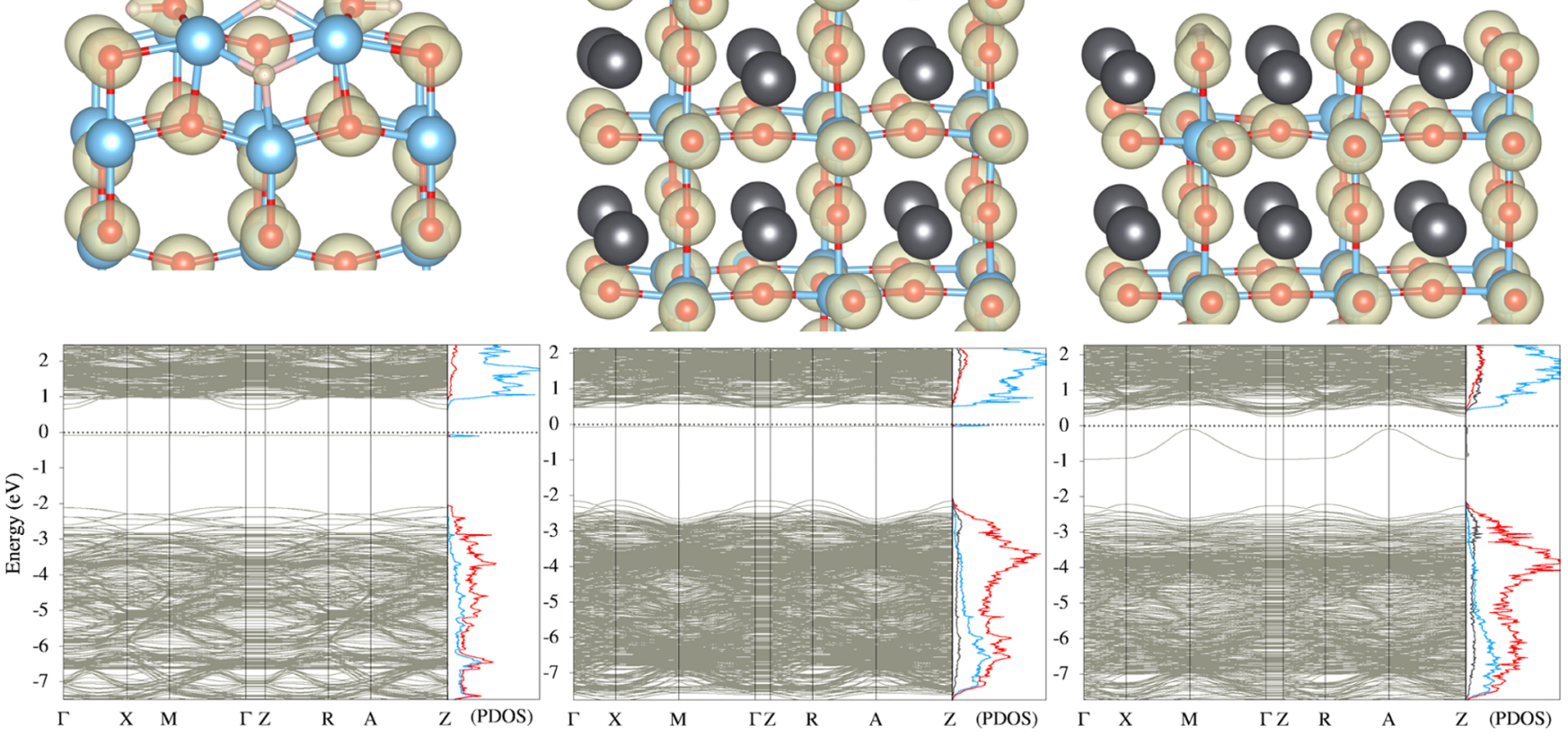

Figure 5. Charge density plots and corresponding electronic band structures with partial densities of states $(\mathrm{PDOS})$ of $(\mathrm{a}) \mathrm{TiO}_{2}(001)-(3 \times 2)+$ $3 \mathrm{O}_{\text {vac }}+2 \mathrm{H}_{2} \mathrm{O}$ system, (b) $\mathrm{PbTiO}_{3}(001)-(3 \times 2)-\mathrm{TiO}_{2}$-terminated $+3 \mathrm{O}_{\text {vac }}+2 \mathrm{H}_{2} \mathrm{O}$ system, and $(\mathrm{c}) \mathrm{PbTiO}_{3}(001)-(3 \times 2)-\mathrm{PbO}$-terminated $+2 \mathrm{O}_{\text {vac }}$ $+2 \mathrm{H}_{2} \mathrm{O}$ system calculated using the SCAN $+U(U=6$ for Ti $3 \mathrm{~d}$ and $U=3$ for $\mathrm{O} 2 \mathrm{p})$ approach. A color scheme of dark gray, light blue, red, and white is used for $\mathrm{Pb}, \mathrm{Ti}, \mathrm{O}$, and $\mathrm{H}$, respectively.

with $\mathrm{H}_{2}$ production on the (001) surfaces of neither $\mathrm{TiO}_{2}$ nor $\mathrm{TiO}_{2}$-terminated $\mathrm{PbTiO}_{3}$ for the oxygen vacancy-mediated models considered here. The energy differences from (b) to (c) are $-3.12 \mathrm{eV}\left(\mathrm{PbO}\right.$ termination of $\left.\mathrm{PbTiO}_{3}\right),-2.13 \mathrm{eV}$ $\left(\mathrm{TiO}_{2}\right.$ termination of $\left.\mathrm{PbTiO}_{3}\right)$, and $-3.51 \mathrm{eV}\left(\mathrm{TiO}_{2}\right)$. The only case that can produce $\mathrm{H}_{2}$ appears to be $\mathrm{PbO}$ termination with two adjacent oxygen vacancies that were occupied with two separate water molecules even though the energy difference in the last stage is smaller than the other cases.

Total charge distributions, the energy bands, and the corresponding partial densities of states (PDOS) were calculated, as shown in Figure 5. Charge density plots show the bonding characteristics of $\mathrm{TiO}_{2}$ and $\mathrm{PbTiO}_{3}$ lattice structures. The calculated Bader charges are consistent with the nominal charge states for the lattice cations and anions as $\mathrm{Ti}^{4+}, \mathrm{O}^{2-}$, and $\mathrm{Pb}^{1+}$. More importantly, the charge distribution after water dissociation reveals the bonding of dissociated $\mathrm{H}$ atoms to either to the substrate or to each other to form $\mathrm{H}_{2}$. Especially, the charge around the $\mathrm{H}$ atoms, which were bound to surface $\mathrm{Ti}$ atoms, indicates a partial filling of the $2 \mathrm{~s}$ orbitals.

Energy bands and electronic densities of states were calculated using the SCAN functional with the supplementary Hubbard- $U$ term to better describe the repulsion between strongly correlated $\mathrm{Ti} 3 \mathrm{~d}$ and, to a lesser degree, $\mathrm{O} 2 \mathrm{p}$ electrons. This is one of the methods necessary to overcome the band gap underestimation of the standard XC functionals within the framework of DFT. In addition, the nature of defect-related states must be reasonably defined in the gap. The use of the SCAN functional instead of standard GGA functionals was not enough to get the band gaps of $\mathrm{TiO}_{2}$ and $\mathrm{PbTiO}_{3}$ properly. Similar findings were reported previously by a theoretical study. ${ }^{17}$ The choice of the supplementary empirical $U$ term becomes critical and needs to be validated for these oxide materials in the presence of point defects. After testing several $U$ parameters for $\mathrm{Ti} 3 \mathrm{~d}$ with and without $\mathrm{O} 2 \mathrm{p}$ orbitals, the optimal choice was found as $U=6$ for $\mathrm{Ti} 3 \mathrm{~d}$ and $U$ $=3$ for $\mathrm{O} 2 \mathrm{p}$ states, which not only heals the band gap but also gives a sound description of the defect-related gap states for both of the materials (please see the Supporting Information). In particular, the application of Hubbard- $U$ for $\mathrm{O} 2 \mathrm{p}$ orbitals becomes important for the electronic description of dissociated $\mathrm{H}$ atoms on a cluster of three oxygen vacancies on $\mathrm{TiO}_{2}$ termination of $\mathrm{PbTiO}_{3}$.

The valence band $(\mathrm{VB})$ dominantly reflects the Ti-O bonding character with a contribution from $\mathrm{Pb}-\mathrm{O}$ bonds in the case of $\mathrm{PbTiO}_{3}$. The upper part of $\mathrm{VB}$ is characterized by O $2 p$ electrons, which is common and well known for transition-metal oxides. The conduction band (CB), on the other hand, dominantly contributed by antibonding $\mathrm{Ti} 3 \mathrm{~d}$ states. In the cases of $\mathrm{TiO}_{2}$-terminated surfaces shown in Figure 5a,b, two hydroxyls are bound to two adjacent vacancies. This binding appears as a doubly degenerate state at the top of $\mathrm{VB}$ reflecting $\mathrm{Ti}-\mathrm{O}$ bonding character. In addition, trapping of dissociated $\mathrm{H}$ atoms at the third oxygen vacancy brings a flat going occupied state 0.73 and $0.55 \mathrm{eV}$ below the conduction band minimum $(\mathrm{CBM})$ of $\mathrm{TiO}_{2}$ and $\mathrm{PbTiO}_{3}$, respectively. Well localization of these defect-related states indicates a weak binding of dissociated $\mathrm{H}$ atoms to the vacancy site on the substrate. In the case of $\mathrm{PbO}$ termination of $\mathrm{PbTiO}_{3}$ shown in Figure 5c, the valence band maximum (VBM) is characterized by the hydroxyl-vacancy bond similar to the previous structures. A gap state $0.34 \mathrm{eV}[\Gamma-\mathrm{M}]$ below the CBM appears due to $\mathrm{H}_{2}$. The dispersion of this state along $k_{z}$ is consistent with the $\mathrm{H}-\mathrm{H}$ covalent bond, which lies in the $z$-direction (or [001]) with respect to the surface plane. The SCAN $+U$ method successfully explains and gives insights into 
the origin and nature of the electronic energy band structure of the models, which were constructed to represent water splitting on $\mathrm{TiO}_{2}$ and $\mathrm{PbTiO}_{3}$.

\section{CONCLUSIONS}

The number and clustering of surface oxygen vacancies become critically important for direct water splitting on the (001) surfaces of $\mathrm{TiO}_{2}$ and $\mathrm{PbTiO}_{3}$. The presence of $\mathrm{Pb}$ weakens the binding of lattice oxygens. Therefore, the energy cost of oxygen vacancy formation on $\mathrm{TiO}_{2}$ is higher than that on $\mathrm{PbTiO}_{3}$. The minimum requirement appears to be the adsorption of two water molecules on two nearest-neighbor oxygen vacancy sites separately for the possibility of a subsequent dissociation of their $\mathrm{H}$ atoms that face each other. $\mathrm{TiO}_{2}$-terminated surfaces need a cluster of three oxygen vacancies in which two of them act as water-capturing active sites and the remaining one must be adjacent to both of them and must remain unoccupied. The existence of this third vacancy is necessary for the dissociation of the $\mathrm{H}$ atoms from their parent water molecules yet is detrimental for the formation of $\mathrm{H}_{2}$ since it acts as a trap for dissociated $\mathrm{H}$ atoms. Revelation of this bottleneck is useful in explaining the production of very low quantities of $\mathrm{H}_{2}$ by direct water splitting on $\mathrm{TiO}_{2}$ terminations, which are otherwise known for their excellent catalytic properties. On $\mathrm{PbO}$ termination of $\mathrm{PbTiO}_{3}$, two adjacent oxygen vacancies can capture two water molecules that lean toward each other. Based on the DFT calculations, vacancy-mediated water splitting seems to be possible on $\mathrm{PbO}$ termination. However, hydrogen molecules must overcome the van der Waals attraction toward the surface.

\section{ASSOCIATED CONTENT}

\section{S1 Supporting Information}

The Supporting Information is available free of charge at https://pubs.acs.org/doi/10.1021/acs.jpcc.0c09685.

3D charge density plots and energy band structures calculated using different $U$ parameters for the (001) terminations of $\mathrm{PbTiO}_{3}$ and $\mathrm{TiO}_{2}$ (PDF)

\section{AUTHOR INFORMATION}

\section{Corresponding Authors}

Ersen Mete - Department of Physics, Balikesir University, Balikesir 10145, Turkey; orcid.org/0000-0002-09165616; Email: emete@balikesir.edu.tr

Deniz Uner - Department of Chemical Engineering, Middle East Technical University, Ankara 06800, Turkey; ๑ orcid.org/0000-0001-8585-3691; Email: uner@ metu.edu.tr

\section{Authors \\ Şinasi Ellialtıoğlu - Basic Sciences, TED University, Ankara 06420, Turkey \\ Oguz Gulseren - Department of Physics, Bilkent University, Ankara 06800, Turkey; 이이이.org/0000-0002-7632-0954}

Complete contact information is available at:

https://pubs.acs.org/10.1021/acs.jpcc.0c09685

\section{Notes}

The authors declare no competing financial interest.

\section{ACKNOWLEDGMENTS}

Deniz Uner acknowledges financial support from TUBITAK under grant no. 117M040.

\section{REFERENCES}

(1) Lewis, N. S. Research Opportunities to Advance Solar Energy Utilization. Science 2016, 351, No. aad1920.

(2) Onishi, H. Sodium Tantalate Photocatalysts Doped with Metal Cations: Why are They Active for Water Splitting? ChemSusChem 2019, 12, 1825-1834.

(3) Wang, Z.; Inoue, Y.; Hisatomi, T.; Ishikawa, R.; Wang, Q.; Takata, T.; Chen, S.; Shibata, N.; Ikuhara, Y.; Domen, K. Overall water splitting by $\mathrm{Ta}_{3} \mathrm{~N}_{5}$ nanorod single crystals grown on the edges of $\mathrm{KTaO}_{3}$ particles. Nat. Catal. 2018, 1, 756-763.

(4) Chiang, T. H.; Lyu, H.; Hisatomi, T.; Goto, Y.; Takata, T.; Katayama, M.; Minegishi, T.; Domen, K. Efficient Photocatalytic Water Splitting Using Al-Doped $\mathrm{SrTiO}_{3}$ Coloaded with Molybdenum Oxide and Rhodium-Chromium Oxide. ACS Catal. 2018, 8, 27822788.

(5) Surendranath, Y. M.; Kanan, W.; Nocera, D. G. Mechanistic Studies of the Oxygen Evolution Reaction by a Cobalt-Phosphate Catalyst at Neutral pH. J. Am. Chem. Soc. 2010, 132, 16501-16509.

(6) Calisan, A.; Ogulgonen, C. G.; Kincal, S.; Yilmaz, A.; Uner, D. Finding the optimum between volatility and cycle temperatures in solar thermochemical hydrogen production: $\mathrm{Pb} / \mathrm{PbO}$ pair. Int. J. Hydrogen Energy 2019, 44, 18671-18681.

(7) Calisan, A.; Ogulgonen, C. G.; Yilmaz, A.; Uner, D.; Kincal, S. Steam methane reforming over structured reactors under concentrated solar irradiation. Int. J. Hydrogen Energy 2019, 44, 1868218693.

(8) Fujishima, A.; Honda, K. Electrochemical Photolysis of Water at a Semiconductor Electrode. Nature 1972, 238, 37-38.

(9) Takata, T.; Jiang, J.; Sakata, Y.; Nakabayashi, M.; Shibata, N.; Nandal, V.; Ski, K.; Hisatomi, T.; Domen, K. Photocatalytic water splitting with a quantum efficiency of almost unity. Nature 2020, 581, 411-414.

(10) Kanan, M. W.; Nocera, D. G. In Situ Formation of an OxygenEvolving Catalyst in Neutral Water Containing Phosphate and $\mathrm{Co}^{2+}$. Science 2008, 321, 1072-1075.

(11) Can, E.; Yildirim, R. Data mining in photocatalytic water splitting over perovskites literature for higher hydrogen production. Appl. Catal., B 2019, 242, 267-283.

(12) Uner, D. O.; Pruski, M.; King, T. S. Optimization of the volumetric hydrogen chemisorption technique for dispersions of $\mathrm{Ru} /$ $\mathrm{SiO}_{2}$ catalysts. J. Catal. 1995, 156, 60-64.

(13) Kresse, G.; Joubert, D. From ultrasoft pseudopotentials to the projector augmented-wave method. Phys. Rev. B 1999, 59, 17581775 .

(14) Blöchl, P. E. Projector augmented-wave method. Phys. Rev. B 1994, 50, 17953-17979.

(15) Sun, J.; Ruzsinszky, A.; Perdew, J. P. Strongly constrained and appropriately normed semilocal density functional. Phys. Rev. Lett. 2015, 115, No. 036402.

(16) Gautam, G. S.; Carter, E. A. Evaluating transition metal oxides within DFT-SCAN and SCAN+U frameworks for solar thermochemical applications. Phys. Rev. Mater. 2018, 2, No. 095401.

(17) Erhart, P.; Klein, A.; Åberg, D.; Sadigh, B. Efficacy of the DFT $+U$ formalism for modeling hole polarons in perovskite oxides. Phys. Rev. B 2014, 90, No. 035204.

(18) Mete, E.; Odabaşı, S.; Mao, H.; Chung, T.; Ellialtıŏlu, Ş.; Reimer, J. A.; Gülseren, O.; Uner, D. Double Perovskite Structure Induced by $\mathrm{Co}$ Addition to $\mathrm{PbTiO}_{3}$ : Insights from DFT and Experimental Solid-State NMR Spectroscopy. J. Phys. Chem. C 2019, 123, 27132-27139.

(19) Ünal, H.; Mete, E.; Ellialtıŏlu, Ş. Surface energy and excess charge in $(1 \times 2)$-reconstructed rutile $\mathrm{TiO}_{2}(110)$ from DFT $+U$ calculations. Phys. Rev. B 2011, 84, No. 115407. 
(20) Tang, W.; Sanville, E.; Henkelman, G. A grid-based Bader analysis algorithm without lattice bias. J. Phys.: Condens. Matter 2009, 21, No. 084204.

(21) Eglitis, R. I.; Vanderbilt, D. Ab initio calculations of $\mathrm{BaTiO}_{3}$ and $\mathrm{PbTiO}_{3}$ (001) and (011) surface structures. Phys. Rev. B 2007, 76, No. 155439.

(22) Takagi, H.; Hwang, H. Y. An Emergent Change of Phase for Electronics. Science 2010, 327, 1601-1602.

(23) Xu, B.; Bhawe, Y.; Davis, M. E. Low-temperature, manganese oxide-based, thermochemical water splitting cycle. Proc. Natl. Acad. Sci. U.S.A. 2012, 109, 9260-9264.

(24) Rao, C. N. R.; Dey, S. Solar thermochemical splitting of water to generate hydrogen. Proc. Natl. Acad. Sci. U.S.A. 2017, 114, 1338513393.

(25) Wendt, S.; Matthiesen, J.; Schaub, R.; Vestergaard, E. K.; Lægsgaard, E.; Besenbacher, F.; Hammer, B. Formation and Splitting of Paired Hydroxyl Groups on Reduced $\mathrm{TiO}_{2}(110)$. Phys. Rev. Lett. 2006, 96, No. 066107.

(26) Petrik, N. G.; Kimmel, G. A. Reaction Kinetics of Water Molecules with Oxygen Vacancies on Rutile $\mathrm{TiO}_{2}(110)$. J. Phys. Chem. C 2015, 119, 23059-23067.

(27) Wang, Z.; Hao, X.; Gerhold, S.; Novotny, Z.; Franchini, C.; McDermott, E.; Schulte, K.; Schmid, M.; Diebold, U. Water Adsorption at the Tetrahedral Titania Surface Layer of $\mathrm{SrTiO}_{3}(110)-(4 \times 1)$. J. Phys. Chem. C 2013, 117, 26060-26069.

(28) Tan, S.; Feng, H.; Zheng, Q.; Cui, X.; Zhao, J.; Luo, Y.; Yang, J.; Wang, B.; Hou, J. G. Interfacial Hydrogen-Bonding Dynamics in Surface-Facilitated Dehydrogenation of Water on $\mathrm{TiO}_{2}(110)$. J. Am. Chem. Soc. 2020, 142, 826-834.

(29) Çakır, D.; Gülseren, O.; Mete, E.; Ellialtıŏlu, Ş. Dye adsorbates BrPDI, BrGly, and BrAsp on anatase $\mathrm{TiO}_{2}(001)$ for dye-sensitized solar cell applications. Phys. Rev. B 2009, 80, No. 035431.

(30) Ünal, H.; Gunceler, D.; Gülseren, O.; Ellialtıŏ̆lu, Ş.; Mete, E. Anatase $\mathrm{TiO}_{2}$ nanowires functionalized by organic sensitizers for solar cells: A screened Coulomb hybrid density functional study. J. Appl. Phys. 2015, 118, No. 194301.

(31) Glezakou, V. A.; Rousseau, R. Shedding light on black titania. Nature Materials 2018, 17, 851-859.

(32) Selcuk, S.; Zhao, X.; Selloni, A. Structural evolution of titanium dioxide during reduction in high-pressure hydrogen. Nat. Mater. 2018, 17, 923-928. 